\title{
A Discrete Model of the Evanescent Light Emission from Ultra-Thin Layers
}

\author{
N. Mirchin ${ }^{*}$, E. Tannous ${ }^{1}$, I. Lapsker ${ }^{2}$, A. Laihtman² ${ }^{2}$ A. Peled ${ }^{1}$ \\ ${ }^{1}$ Electronic Engineering Department, Holon Institute of Technology, Holon, Israel \\ ${ }^{2}$ Science Department, Holon Institute of Technology, Holon, Israel \\ Email: ${ }^{*}$ nina.mirchin@gmail.com
}

Received 27 February 2015; accepted 1 May 2015; published 7 May 2015

Copyright (C) 2015 by authors and Scientific Research Publishing Inc.

This work is licensed under the Creative Commons Attribution International License (CC BY).

http://creativecommons.org/licenses/by/4.0/

(c) (i) Open Access

\begin{abstract}
A discrete model of the Differential Evanescent Light Intensity (DELI) technique was developed to calculate and map 3D nanolayers thicknesses from the evanescent light intensity captured from optical waveguides. The model was used for ultra-thin Pd nanometric layers sputtered on glass substrates. The layers thickness profiles were displayed in 3D and 1D profiles plots. The total thickness profiles of the ultra-thin Pd films obtained in the range of $1-10 \mathbf{~ n m}$ were validated using AFM measurements. Based on the model developed the evanescent photon extraction parameter of the material was estimated.
\end{abstract}

\section{Keywords}

Evanescent Waves, Light Scattering, Thin Nanofilms, Thickness Profiles, Optical Nanoscopy

\section{Introduction}

The investigation of optical scattering from nanostructures shows a growing interest due to current trends for novel nanometric surface analysis techniques. A useful phenomenon in this endeavour is the evanescent light interaction with nanofilms deposited on a surface. Many models have been proposed to describe the phenomenon of evanescent waves in nano-optics but at present there is still no conclusive physical picture for it [1]-[9]. More experimental data in this field becomes thus of both theoretical and practical interest.

The optical microscopy technique named Differential Evanescent Light Intensity (DELI) was used in the past decade to investigate nanostructures profiles of various materials films with small thicknesses obtaining information also about the optical photon scattering parameters [10]-[16]. The DELI technique is based on the phe-

\footnotetext{
${ }^{*}$ Corresponding author.
}

How to cite this paper: Mirchin, N., Tannous, E., Lapsker, I., Laihtman, A. and Peled, A. (2015) A Discrete Model of the Evanescent Light Emission from Ultra-Thin Layers. Journal of Materials Science and Chemical Engineering, 3, 30-36.

http://dx.doi.org/10.4236/msce.2015.35004 
nomenon of Total Internal Reflection (TIR) [4] [5] at interfaces where evanescent waves and optical tunnelling may occur when peculiar boundary conditions are given. Due to its excellent optical contrast vs. dark background, the DELI technique provides convenient nanometre thickness z-profiling using a rather simple optical microscopy densitometry technique, as compared to SEM or TEM microscopies which require complex and expensive electron beam systems and vacuum chambers. The DELI surface morphology observation technique is also much easier to perform, faster, and non-destructive for nanometre films profiling as compared to electron beam microscopy (EBM) or even the simpler atomic force microscopy (AFM). It is also better suited for nanostructures morphology mapping of large areas as required for instance in industrial applications [10]-[16].

In this work we describe a discrete model for DELI which in addition to the evanescent phenomenon takes into account also the optical absorption. Then this discrete model is utilized to analyse and construct the thickness profiles of sputtered Pd films with ultra-thin thicknesses in the range of 1 - $10 \mathrm{~nm}$ and the results are compared in Section 3 with the previously used model in Ref. [15].

\section{General DELI Theory}

In the past, nanometric thin films of various materials [10]-[16] and with thicknesses in the range of $1-200 \mathrm{~nm}$ were deposited on top of glass substrates which also served as light waveguides. Then, they were observed from the top by an optical microscope, obtaining the 3D spatial profiles and characteristic parameters of the evanescent field using the DELI phenomenological model [10]-[13]. To evaluate the relative and true surface nanoprofile thicknesses of the various samples from the evanescent captured images, the mean Normalized Integrated Optical Density (NIOD), defined in Equation (1) is obtained experimentally from the evanescent optical density of images from the deposited zones:

$$
N I O D=\frac{1}{S} \iint_{S} D(x, y) \cdot \mathrm{d} s=\frac{1}{S} \int_{0}^{\infty} D \cdot H(D) \mathrm{d} D
$$

where $D(x, y)$ is the gray level value of each pixel $(0-255), S$ is the sampled image area and $H(D)$ is the image histogram i.e., the number of pixels per gray level. In this work, to convert the NIOD to absolute thickness values we used the known sputtering rates to calculate the mean thicknesses of the Pd samples. From AFM surface scans, see Figure 1(c) and the experimental sputtering rate the mean thickness of the thickest Pd sample was estimated as $\sim 10 \mathrm{~nm}$.

\section{A DELI Discrete Model}

We present here a discrete model for the captured evanescent light intensity. Let the nanometric film of thickness $h$ be discretized into $N$ layers ( $n=1,2, N$ ), each with a thickness equal to the crystalline unit cell dimension $a$ of the deposited material. It can be considered that in every $n^{\text {th }}$ layer, the evanescent light intensity decreases exponentially in the z-direction. Assuming that the light beam is scattered by the atoms in the nano layers, the perpendicular light intensity scattered by the $n^{\text {th }}$ layer can be described by:



(a)

(b)

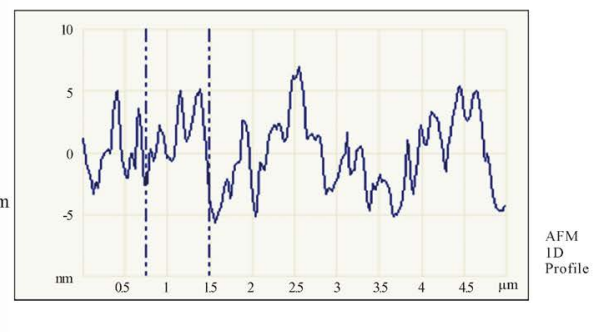

(c)

Figure 1. 3D perspective and 1D profile from a sampled area with a mean thickness of $\sim 10$ (nm): (a) DELI image of an (520 $\times 520) \mu \mathrm{m}^{2}$ sampled area; (b) AFM image of an $(5 \times 5) \mu \mathrm{m}^{2}$ sampled area and (c) 1D-AFM sample thickness profile of (b). Note the different scale of images (a) and (b). 


$$
I_{n}=N_{a} \cdot a \cdot \sigma_{e w} \cdot I_{0} \cdot \mathrm{e}^{-\gamma \cdot n \cdot a}
$$

where $N_{a}$ is the deposited material atom volume density, in $\left(\mathrm{cm}^{-3}\right), a$ is the crystalline unit cell dimension assumed identical for every layer, in $\mathrm{cm}$, and $\sigma_{e w}$ is the evanescent extraction cross section of each atom, in $\left(\mathrm{cm}^{2}\right) . I_{0}$ denotes the longitudinal propagating optical field intensity below the waveguide/material interface and $\gamma=2 / d_{m}$ is the evanescent photon extraction parameter of the material, where $d_{m}$ is an "effective" evanescent depth. To simplify matters, we assume that $\gamma(h)$ is constant at every $h$.

Considering every layer as a local source of scattered light and neglecting multiple scattering we obtain the following expression for the upward evanescent scattered integrated light intensity from a layer of overall thickness $h=N \cdot a$, taking also into account the optical absorption coefficient $\alpha$ of the material:

$$
I(h=N a)=\sum_{n=1}^{N} \frac{1}{2} \cdot I_{n} \mathrm{e}^{-\alpha \cdot(N-n) \cdot a}
$$

Here we assumed that in the far field case, only half of the total scattered intensity from the layer propagates in the upward direction as obtained in Ref. [3]. Using (2) in (3) and denoting a scattering parameter by $K(x, y, z)=N_{a}(x, y, z) \cdot \sigma_{e w}(x, y, z)$, we obtain:

$$
I(h)=\frac{1}{2} \cdot K \cdot a \cdot I_{0} \cdot \mathrm{e}^{-\alpha \cdot h} \sum_{n=1}^{N} \mathrm{e}^{(\alpha-\gamma) \cdot n \cdot a}=\frac{1}{2} \cdot K \cdot a \cdot I_{0} \cdot \mathrm{e}^{-\alpha \cdot h} \cdot S_{N}
$$

where $S_{N}$ denotes the sum of $N$ terms of a geometrical series $S_{N}=\sum_{n=1}^{N} a_{n}=\sum_{n=1}^{N} q^{n}$, i.e., $S_{N}=\frac{a_{1}-a_{N} q}{1-q}$, with
$a_{1}=\mathrm{e}^{(\alpha-\gamma) a}, \quad a_{N}=\mathrm{e}^{(\alpha-\gamma) a N}$ and $q=\mathrm{e}^{(\alpha-\gamma) a}:$

$$
S_{N}=\sum_{n=1}^{N} \mathrm{e}^{(\alpha-\gamma) \cdot n \cdot a}=\frac{\left(1-\mathrm{e}^{(\alpha-\gamma) h}\right)}{\left[\mathrm{e}^{-a(\alpha-\gamma)}-1\right]}
$$

From Equation (4) and Equation (5) the normalized evanescent light intensity defined as a fractional scattered evanescent intensity $\eta$, is given by:

$$
\eta(h)=\frac{I(h)}{I_{0}}=\frac{1}{2} \cdot K \cdot a \cdot \frac{\left(\mathrm{e}^{-\alpha \cdot h}-\mathrm{e}^{\gamma \cdot h}\right)}{\left(\mathrm{e}^{-(\alpha-\gamma) a}-1\right)}
$$

For two layers with thicknesses $h_{1}, h_{2}$ we obtain from Equation (6):

$$
\eta_{1}\left(h_{1}\right)=\frac{1}{2} \cdot K \cdot a \cdot \frac{\left(\mathrm{e}^{-\alpha \cdot h_{1}}-\mathrm{e}^{-\gamma \cdot h_{1}}\right)}{\left(\mathrm{e}^{-(\alpha-\gamma) a}-1\right)} \text { and } \eta_{2}\left(h_{2}\right)=\frac{1}{2} \cdot K \cdot a \cdot \frac{\left(\mathrm{e}^{-\alpha \cdot h_{2}}-\mathrm{e}^{-\gamma \cdot h_{2}}\right)}{\left(\mathrm{e}^{-(\alpha-\gamma) a}-1\right)}
$$

We also assume that the experimentally measured NIOD is proportional to $\eta$ from Equations (6) and (7), hence the ratio $k$ for two NIOD's and $\eta_{i}, i=1,2$ for two thicknesses $h_{1}$, and $h_{2}$ is given approximately by:

$$
k\left(\gamma, h_{1}, h_{2}\right)=\frac{\operatorname{NIOD}_{2}\left(h_{2}\right)}{\operatorname{NIOD}_{1}\left(h_{1}\right)} \approx \frac{\eta_{2}}{\eta_{1}}=\frac{\left(\mathrm{e}^{-\alpha h_{2}}-\mathrm{e}^{-\gamma h_{2}}\right)}{\left(\mathrm{e}^{-\alpha h_{1}}-\mathrm{e}^{-\gamma h_{1}}\right)}
$$

Thus, given the absolute thickness $h_{2}$ at one point we can calculate also other thicknesses values $h_{i}$ of the nanoprofile from Equation (8) provided $\gamma$ is known and $k$ is obtained from the optical density measurements of NIOD [10]-[13]. For absolute thicknesses values determination, the average layers thicknesses can be calibrated by independent techniques such as material deposition rates, spectrometry, SEM, AFM or mechanical nanoprofilometry.

The evanescent photon extraction parameter $\gamma$ of the material and the "effective" thickness parameter $d_{m}=2 / \gamma$ can be determined from the NIOD experimental data and the theoretical model of Equation (6). Equation (8) is a transcendental equation, i.e., analytically unsolvable. One approximation procedure to calculate 
$\gamma$ from Equation (8) is to measure any two NIOD for known thicknesses $h_{2}>h_{1}$ where $\mathrm{NIOD}_{2}>\mathrm{NIOD}_{1}$, and using the optical absorption constant $\alpha$ for the material. In our case for Pd, $\alpha=0.091 \mathrm{~nm}^{-1}$ is calculated from $\alpha=4 \pi k_{2} / \lambda$ [18] using the complex value of the dielectric parameter $k_{2}$ of Palladium thin films given in Ref. [19] at $\lambda=555 \mathrm{~nm}$. From the experimental observation (see Figure 2) it follows that the scattered light intensity captured from thicker layers is greater than that from the thinnest.

For $h_{2}=10 \mathrm{~nm}$ and $h_{1}=1 \mathrm{~nm}$ we obtain the experimental ratio $k=126 / 18 \sim 7$, hence from Equation (8) we have:

$$
k=\frac{\left(\mathrm{e}^{-0.91}-\mathrm{e}^{-10 \gamma}\right)}{\left(\mathrm{e}^{-0.091}-\mathrm{e}^{-\gamma}\right)} \approx 7
$$

A solution of Equation (9) can be obtained considering that the term $\mathrm{e}^{-10 \gamma}$ can be neglected as $\gamma \gg 0.091$. In this case the solution of Equation (9) gives $\gamma=0.156 \mathrm{~nm}^{-1}$. We can use this approach to solve Equation (9) because the ratio of the omitted term i.e., $\mathrm{e}^{-10 \gamma}$ with respect to $\mathrm{e}^{-\gamma}$ for all positive $\gamma$ is smaller than $1 / 7=14 \%$. This can also be used as an estimation of the error $\delta$ in this approximation: $\delta \approx \mathrm{e}^{-10 \gamma} / \mathrm{e}^{-\gamma}=\mathrm{e}^{-\gamma \cdot(10-1)} / 7=\mathrm{e}^{-9 \gamma} / 7$. Using the value for $\gamma$ obtained above, it is about $\delta \propto \mathrm{e}^{-9 \gamma} / 7=\mathrm{e}^{-0.156} / 7 \approx 0.035 \approx 3.5 \%$.

Another approximate numerical solution of Equation (9) can be sought for the case $\gamma h_{2}<1$. Using here only the first four terms in the series expansion $\mathrm{e}^{-x} \approx 1-x+\frac{x^{2}}{2 !}-\frac{x^{3}}{3 !}+\cdots$ we obtain for $\gamma$ the equation: $\gamma^{3}-0.281 \gamma^{2}+0.018 \gamma+6.95 \times 10^{-5}=0$. The only solution for this equation making sense physically (due to $\gamma>$ 0 ) is $\gamma \approx 0.0875 \mathrm{~nm}^{-1}$.

We check next the validity of the two different values comparing them to the experimental data obtained from the NIOD. For comparison we used the normalized scattered intensity function for $\eta(h)$ from Equation (6), defined by the ratio of the function to its maximum, i.e., $I_{\text {norm }}(h)=\eta / \eta_{\max }$, where $\eta_{\max }=\kappa(1+\kappa)^{-\left(1+\kappa^{-1}\right)}$, (with $\kappa=\gamma / \alpha)$, is the maximum value $\eta\left(h_{\max }\right)$ at a thickness of $h_{\max }=\ln (\alpha / \gamma) /(\alpha-\gamma)$. This function is plotted in Figure 3 using the two values obtained above, i.e., $\gamma=0.156 \mathrm{~nm}^{-1}$ for curve (a), and $\gamma \approx 0.0875 \mathrm{~nm}^{-1}$ for curve (b). The continuous curves (a) and (b) in Figure 3 show a maxima at the thickness given by $h_{\max }=\ln (\alpha / \gamma) /(\alpha-\gamma)$, giving for $\gamma=0.156 \mathrm{~nm}^{-1}$ the value $h_{\max }=8.3 \mathrm{~nm}$ and for $\gamma \approx 0.0875 \mathrm{~nm}^{-1}$ the value $h_{\max }=11.1 \mathrm{~nm}$.

The asterisks in Figure 3 are the experimental points from the Palladium films optical densitometry data

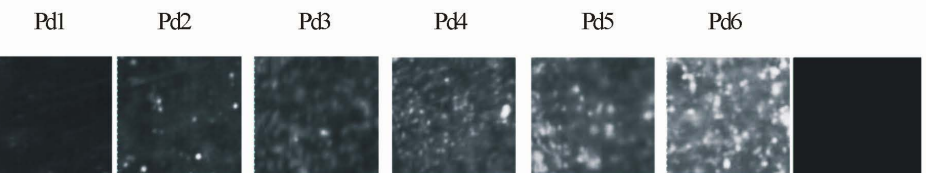

$2 \mathrm{D}$ images

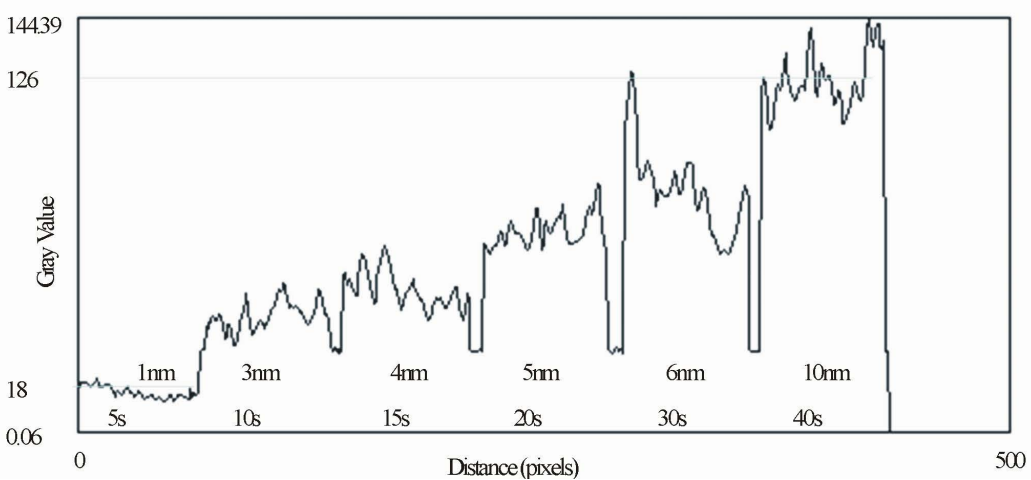

Figure 2. 2D images of $6 \mathrm{Pd}$ sampled areas with mean thicknesses in the range $1-10 \mathrm{~nm}$. The sampled 2D zones have an area of $(520 \times 520) \mu m^{2}$. Below the 2D images, the optical density gray value of a $1 \mathrm{D}$ profile plot averaged across the sampled areas is shown. The last black square is the control zero intensity value. 
obtained for the NIOD's from Figure 2. The highest NIOD obtained is for the greatest thickness point obtained from the sputtering rates measurements giving $h_{1}=10 \mathrm{~nm}$, corresponding well with the AFM profilometry, see Figure 1(c). Since curve (a) is clearly a better fit for the experimental points, we can assume that the value $\gamma=0.156 \mathrm{~nm}^{-1}$ is the more acceptable result for the evanescent photon extraction parameter $\gamma$ of the material. In [15] we obtained in a simpler model without absorption being taken into account giving $\gamma=0.1 \mathrm{~nm}^{-1}$. We also observe in this paper that $\gamma$ is larger than the absorption coefficient, which is $\alpha=0.091 \mathrm{~nm}^{-1}$.

\section{Discussion}

Regarding the mechanism of the evanescent wave extraction, many investigators assume that the material molecules dipoles deposited on the waveguide interface are responsible for the evanescent photons scattering [1]-[9]. The maximum number of dipole molecules per unit volume $N_{a}$ can be estimated in a simple model by the following equation [18]:

$$
N_{a}=\frac{N_{0} \rho}{M}
$$

where $N_{0}=6.022 \times 10^{23}$ [atoms $/ \mathrm{mol}$ ] is the Avogadro number, $\rho$ is the material density in $\left[\mathrm{gr} / \mathrm{cm}^{3}\right.$ ] and $M$ is the material molecular mass in $[\mathrm{gr} / \mathrm{mol}]$. For Pd $\rho=12.02 \mathrm{gr} / \mathrm{cm}^{3}$ and $M=106.42 \mathrm{gr} / \mathrm{mol}$, so that $N_{a}=0.68 \times$ $10^{23}$ atoms $/ \mathrm{cm}^{3}$.

The material optical absorption coefficient $\alpha$ is usually related to the optical absorption cross-section $\sigma_{a}$ and the concentration of the light absorbing molecules $N_{a}$ by the following equation:

$$
\alpha=\sigma_{a} \cdot N_{a}
$$

Analogously, $\gamma$ can be also considered as a product of the volume concentration of the light scattering dipoles $N_{a}$, each with an effective evanescent scattering cross-section $\sigma_{e w}$ given by:

$$
\gamma=\sigma_{e w} \cdot N_{a}
$$

Then, using Equation (12) and the number density of molecule dipoles per unit volume obtained from Equation (10), we obtain an estimation for the "effective" evanescent scattering cross-section of the Pd nanofilms molecules $\sigma_{e w}=1.287 \times 10^{-9} \mu \mathrm{m}^{2}$.

In reference [2], typical optical scattering properties of Ag nano-particles deposited on a substrate were inves-

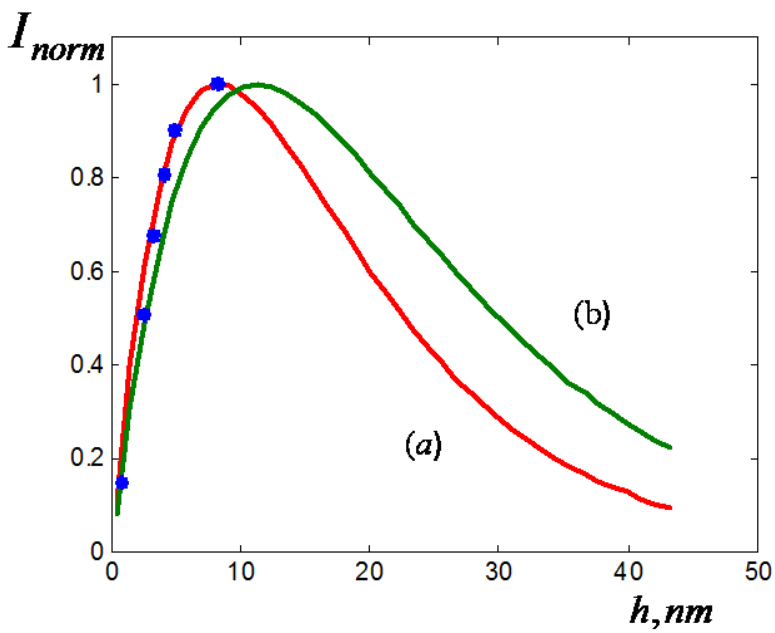

Figure 3. Plots of $I_{\text {norm }}(h)=\eta / \eta_{\max }$ from Equation (8) for the Pd nanolayer vs. mean thickness of the nanolayer $h$ : (a) for $\gamma=0.156 \mathrm{~nm}^{-1}$ and (b) for $\gamma=0.0875 \mathrm{~nm}^{-1}$. The Pd parameters used were: $a=0.389 \mathrm{~nm}$ [17] and $\alpha=0.091 \mathrm{~nm}^{-1}$ [18]. The asterisks are the experimental NIOD data points. 
tigated by the discrete source method. The numerical evaluations for Ag spheres with diameter $\mathrm{D}=48 \mathrm{~nm}$ on Ag films of thickness $d=49 \mathrm{~nm}$ irradiated with $\lambda=532 \mathrm{~nm}$ light for incident angles $\theta_{i}$ near $90^{\circ}$, gave light scattering cross sections $\sigma_{s c}$ of about $10^{-9} \mu \mathrm{m}^{2}$. Although the metal is different in [2], this result is quite comparable to our results. This strengthens our assumption that optical scattering from the deposited nanoparticles is the mechanism for the extraction of the evanescent waves by the material on top of the waveguide.

The results of evanescent experiments [10]-[16] including the current work for extremely thin layers of Pd in the range 1 - $10 \mathrm{~nm}$ show that such layers located in the evanescent field zone contribute to a sensitive observation of optical density variation due to thickness variations, which can be recorded easily using optical microscopes equipped with a camera. The contribution of the evanescent waves due to the scattered field was discussed in detail in [1] and it has been shown by the method of angular spectrum representation that for a scattering medium which is located near the sources (up to a distance of the light half wavelength $\lambda / 2$ ), the evanescent field becomes an observable optical field [1].

Also, far field scattering optical spatial distributions for different polarized evanescent waves from particles with several sizes were calculated in [3]. For the case $\beta r<0.1, \beta$ being the wavenumber, i.e., when the particle radius is $r<0.1 \lambda / 2 \pi \approx 8 \mathrm{~nm}$, it was shown that for an s-polarized incident wave the scattering diagram in the space in the direction perpendicular to the interface between the two media has a maximum in the $+\mathrm{z}$ direction and one in the $-\mathrm{z}$ direction. This suggests that approximately only half of the scattered intensity goes upward as assumed in Equation (3).

\section{Conclusion}

A discrete layers evanescent model was developed to evaluate the evanescent light extraction parameter $\gamma$ for Pd ultra-thin nanolayers with thicknesses below $10 \mathrm{~nm}$. The experimental z-profiling optical microscopy method called DELI, confirmed to be extremely sensitive, enabling simple and effective optical microscopy observation of thickness variations in ultra-thin Pd films in the range of 1 - $10 \mathrm{~nm}$.

\section{References}

[1] Tong, Z. and Korotkova, O. (2012) Contribution of Evanescent Incident Waves to the Scattered Far Field. Physical Review A, 85, 04382. http://dx.doi.org/10.1103/PhysRevA.85.043802

[2] Grishina, N.V., Eremin, Yu.A. and Sveshnikopv, A.G. (2011) Study of Extraordinary Scattering of Evanescent Waves by the Discrete Sources Method. Computational Mathematics and Mathematical Physics, 51, 1605-1613. http://dx.doi.org/10.1134/S0965542511090119

[3] Bekshaev, A.Y., Bliokh, K.Y. and Nori, F. (2013) Mie Scattering and Optical Forces from Evanescent Fields: A Complex-Angle Approach. Optic Express, 21, 7082-7095. http://dx.doi.org/10.1364/OE.21.007082

[4] Novotny, L. and Hecht, B. (2006) Principles of Nano-Optics. Cambridge University Press, Cambridge, UK.

[5] de Fornel, F. (2000) Evanescent Waves From Newtonian Optics To Atomic Optics, Vol. 73 of Springer Series in Optical Sciences. Springer Verlag, Berlin.

[6] Girard, C. (2005) Near Fields in Nanostructures. Reports on Progress in Physics, 68, 1883-1933. http://dx.doi.org/10.1088/0034-4885/68/8/R05

[7] Eremina, E., Grishina, N., Eremin, Y., Helden, L. and Wriedt, T. (2006) Total Internal Reflection Microscopy with a Multilayered Interface: A Light Scattering Model Based on a Discrete Sources Method. Journal of Optics A: Pure and Applied Optics, 8, 999-1006. http://dx.doi.org/10.1088/1464-4258/8/11/011

[8] Quinten, M., Pack, A. and Wannemacher, R. (1999) Scattering and Extinction of Evanescent Waves by Small Particles. Applied Physics B, 68, 87-92. http://dx.doi.org/10.1007/s003400050591

[9] Wiederrecht, G.P. (2004) Near-Field Optical Imaging of Noble Metal Nanoparticles. The European Physical JournalApplied Physics, 28, 3-18. http://dx.doi.org/10.1051/epjap:2004170

[10] Socol, G., Axente, E., Oane, M., Voicu, L., Petris, A., Vlad, V., Mihailescu, I.N., Mirchin, N., Margolin, R., Naot, D. and Peled, A. (2007) Nanoscopic Photodeposited Structures Analyzed by an Evanescent Optical Method. Applied Surface Science, 253, 6535-6538. http://dx.doi.org/10.1016/j.apsusc.2007.01.076

[11] Mirchin, N., Gankin, M., Gorodetsky, U., Popescu, S.A., Lapsker, I., Peled, A., Duta, L., Dorcioman, G., Popescu, A. and Mihailescu, I.N. (2010) Estimation of Polyethylene Nanothin Layer Morphology by Differential Evanescent Light Intensity Imaging. Journal of Nanophotonics, 4, Article ID: 014760. http://dx.doi.org/10.1117/1.3477983

[12] Mirchin, N., Apter, B., Lapsker, I., Fogel, V., Gorodetsky, U., Popescu, S.A., Peled, A., Popescu-Pelin, G., Dorcioman, 
G., Duta, L., Popescu, A. and Mihailescu, I.N. (2012) Measuring Nanolayer Profiles of Various Materials by Evanescent Light Technique. Journal of Nanoscience and Nanotechnology, 12, 2668-2671. http://dx.doi.org/10.1166/jnn.2012.5788

[13] Mirchin, N. and Peled, A. (2012) Evaluating Nanoprofiles of Materials Deposited on Waveguides by an Evanescent Light Method. Journal of Advanced Microscopy Research, 7, 26-31(6). http://dx.doi.org/10.1166/jamr.2012.1094

[14] Mirchin, N., Peled, A., Duta, L., Popescu, A.C., Dorcioman, G. and Mihailescu, I.N. (2013) Nanoprofiles Evaluation of ZnO Thin Films by an Evanescent Light Method. Microscopy Research and Technique, 76, 992-996. http://dx.doi.org/10.1002/jemt.22259

[15] Mirchin, N., Lapsker, I., Tannous, E. and Peled, A. (2013) Palladium Ultra Thin Layer Profiles Evaluation by Evanescent Light. Materials Sciences and Applications, 4, 572-577. http://dx.doi.org/10.4236/msa.2013.49070

[16] Mirchin, N., Peled, A., Azoulay, J., Duta, L., Dorcioman, G., Popescu, A.C. and Mihailescu, I.N. (2014) Nanoprofiles of $\mathrm{TiO}_{2}$ Films Deposited by PLD Using an Evanescent Light Method. World Journal of Engineering, 11, 111-116. http://dx.doi.org/10.1260/1708-5284.11.2.111

[17] Generalic, Eni. (28 September 2013) Palladium. EniG. Periodic Table of the Elements. KTF-Split. http://www.periodni.com/pd.htm

[18] Hummel, R.E. (2001) Electronic Properties of Materials. 3rd Edition, Springer-Verlag Inc., New York, 204. http://dx.doi.org/10.1007/978-3-642-86538-1

[19] Sullivan, B.T. (1990) Optical Properties of Palladium in the Visible and Near UV Spectral Regions. Applied Optics, 29, 1964-1970. http://dx.doi.org/10.1364/AO.29.001964 